\title{
Brussels Griffon
}

National Cancer Institute

\section{Source}

National Cancer Institute. Brussels Griffon. NCI Thesaurus. Code C53925.

The Griffon is a sturdy toy dog of square proportions with a domed head, undershot jaw, and a very short nose. There are two types: Rough Coated (harsh wiry and dense), and Smooth-Coated or Petit Brabancon variety (short, straight, glossy). Griffons come in red, a mixture of red-brown and black, black and tan, and solid black. Height: 7-8 inches (18$20 \mathrm{~cm}$.) Weight: 6-12 pounds (2.5-5.5 kg.) 